\title{
EL CORPUS COMO HERRAMIENTA PARA LA TRADUCCIÓN ESPECIALIZADA ITALIANO/ESPAÑOL: UNA EXPERIENCIA CONTEXTOS DE LA INDUSTRIA COSMÉTICA ${ }^{1}$
}

\author{
Estefanía Flores Acuña \\ Universidad Pablo de Olavide (Sevilla)
}

\begin{abstract}
Resumen: En este trabajo se presentan algunas posibilidades del corpus como fuente de información documental, terminológica y textual para la traducción especializada italiano/español. Proporcionamos pautas para la compilación y explotación en clase de un corpus ad hoc con el objetivo de que el alumno aprenda a recopilar rápidamente documentación fiable que le ayude a afrontar con mayor seguridad y garantías de éxito un encargo de traducción italiano/ español en un campo específico como la cosmética. En esta combinación lingüística, además, la escasez de recursos lexicográficos impresos y electrónicos justifica aún más la necesidad de aprender a elaborar una herramienta flexible, de bajo coste y de gran valor para el traductor profesional.
\end{abstract}

Palabras clave: traducción especializada, lingüística de corpus, cosmética, didáctica de la traducción, lengua italiana.

Abstract: This article examines some uses of corpora as efficient sources of terminological, textual and conceptual information. By compiling an ad hoc specialised corpus, students will have access to a variety of documents which will allow them to translate from Italian into Spanish more confidently and more successfully, especially in a specialised area such as cosmetics. Unfortunately, lexicographic resources for the language combination Italian-Spanish are not as numerous as those including other languages, particularly when it comes to specialised lexicography. That is why this article highlights the need of designing a flexible, low-cost tool -yet essential- for professional translators. Keywords: specialised translation, corpus linguistics, cosmetics, didactics of translation, Italian language.

${ }^{1} \mathrm{El}$ presente trabajo ha sido realizado (parcialmente) en el seno del proyecto 'TRADICOR: Sistema de gestión de corpus para la innovación didáctica en traducción e interpretación' (PIE 13-054). 


\section{INTRODUCCIÓN}

No hay época o cultura en que no haya existido una mayor o menor preocupación del ser humano por su imagen y bienestar. El discurso en torno a la belleza y al cuidado del cuerpo está muy lejos de ser construido a la ligera o con elementos elegidos al azar. Tanto es así que numerosos estudios sobre "la retórica del bienestar" (Ventura 2000; Díaz Rojo et al. 2006; Morant Marco y Martín 2010) demuestran cómo es precisamente este discurso el culpable en muchos casos de la obsesión de tantos hombres y mujeres por ajustarse al canon estético imperante en la sociedad occidental actual.

$\mathrm{E} 1$ ansia por conseguir esta imagen ideal -y pocas veces alcanzable- conduce a un consumo continuo de productos y tratamientos cosméticos que prometen acercarnos a esta meta casi sin esfuerzo. ${ }^{2}$ Tanto es así que en nuestro país, por ejemplo, uno de los tres pilares del consumo es el cuidado personal, junto con la alimentación y el cuidado del hogar. ${ }^{3}$ En efecto, pese a la crisis económica actual, el mercado español mantiene su quinta posición en Europa por volumen de facturación. ${ }^{4}$ Además, la cifra de exportación en este sector representa la nada despreciable cifra del 1,2\% del total nacional.

Italia, por su parte, es el cuarto mercado europeo en el sector de la cosmética, después de Alemania, Francia y Reino Unido. En poco más de veinte años, la industria cosmética italiana se ha consolidado en los mercados extranjeros, registrando en sus exportaciones un crecimiento medio anual superior al 5\%. Entre los países destinatarios de estas exportaciones, España ocupa el quinto lugar (después de Alemania, Francia, Reino Unido y Estados Unidos). ${ }^{5}$

Actualmente, el concepto de belleza es indisociable del de salud. Ser bellos equivale hoy en día a estar sanos: dietas, cosméticos, tratamientos estéticos... todo nos conduce a la meta de un bienestar que nos proporcione simultáneamente un aspecto saludable y atractivo. De ahí que se haya llegado a hablar de la medicalización de la publicidad de los cosméticos, que se presentan como productos pseudosanitarios (Díaz Rojo, 2001-2002). Para transmitir esta idea de rigor, innovación y eficacia, el lenguaje de la industria cosmética se impregna con frecuencia de un vocabulario pseudocientífico plagado de tecnicismos, extranjerismos, siglas, prefijos, sufijos, compuestos, referencias al mundo de la medicina, la botánica y la naturaleza...

El abanico de géneros textuales que suelen ser objeto de traducción en este sector es muy amplio: etiquetas de productos, material informativo para

2 Es el silenciamiento del sacrificio en la retórica del culto a la belleza y a la salud (Díaz Rojo et al. 2006: 201).

${ }^{3}$ Según declaraciones de Esteban Rodés, presidente de Stanpa (Asociación Nacional de Perfumería y Cosmética).

${ }^{4}$ Detrás de Alemania, Francia, Inglaterra e Italia.

5 Véase informe Unipro junio 2013 http://www.unipro.org/home/it/documenti/centro_studi/ beauty_report_2013/MATTINO_def_-_short.pdf 
ventas, textos publicitarios, sitios web, documentos legales, patentes, trabajos científicos, etc. Teniendo en cuenta las variables temática, usuarios y situación de comunicación, nos encontramos en muchos de estos géneros ante un auténtico discurso especializado para cuya traducción se requieren competencias y habilidades específicas relativas a la documentación necesaria, la terminología empleada y las convenciones textuales propias del género en cada cultura.

No en todos los géneros se da el mismo grado de especialización. Es lo que Cabré denomina perspectiva, "l'óptica des de la qual s'aborda en el missatge" (1998: 177). Aunque el emisor sea siempre un especialista que transmite un mensaje sobre su campo de especialización, el destinatario no siempre es un especialista, puede también ser un público semiexperto o profano en la materia. Así por ejemplo, la temática no suele ser tan especializada en los textos publicitarios o en algunas webs informativas, aunque la terminología sea empleada intencionadamente para dar apariencia de alto nivel de competencia.

En las páginas que siguen expondremos cómo compilar y explotar un corpus ad hoc en la clase de traducción especializada. Presentaremos en primer lugar nuestro planteamiento de la asignatura en el Grado de Traducción e Interpretación, repasaremos el panorama actual de recursos documentales y terminológicos existentes para este campo en la combinación italiano/español, reflexionaremos sobre la contribución de la Lingüística de corpus al mundo de la Traducción para centrarnos, por último, en un caso práctico de aplicación de un corpus ad hoc de textos comparables para abordar la traducción del italiano al español de un sitio web sobre cosmética.

2. LA TRADUCCIÓN ESPECIALIZADA ITALIANO/ESPAÑOL EN EL GRADO EN TRADUCCIÓN E INTERPRETACIÓN (TEI)

La Traducción Especializada Italiano/Español en el Grado en Traducción e Interpretación de nuestra universidad se vertebra en dos asignaturas semestrales, Traducción Especializada de la Lengua CI (Italiano) y Traducción Especializada de la Lengua CII (Italiano), de 6 créditos cada una y cursadas en $3^{\circ}$ y $4^{\circ}$ curso respectivamente. Como ya hemos expuesto en anteriores trabajos (Flores Acuña 2012, en prensa), insistimos en la necesidad de que los bloques temáticos que presentamos en estas asignaturas respondan a la realidad del mercado laboral en nuestra combinación lingüística. Esto no solo aumenta la motivación del alumnado, sino que permite que muchos de los encargos planteados en clase sean reales, pues corresponden a encargos recibidos en nuestra actividad profesional paralela a la docencia.

Los campos temáticos que los alumnos han de trabajar en ambas asignaturas son los siguientes: 1) Diseño y montaje; 2) Moda; 3) Belleza y cosmética; 4) Derecho; 5) Economía, formación y empleo; 6) Humanidades. Procuramos que el contenido de cada texto de un mismo bloque temático sea diferente, por lo 
que el proceso de documentación comienza desde el principio con cada nuevo encargo, tal y como ocurre con frecuencia en la realidad profesional.

Dedicamos todo un bloque de nuestra programación al mundo de la belleza y la cosmética, habida cuenta del creciente número de empresas italianas de este sector que solicitan la traducción al español de sus catálogos y de sus sitios web. Téngase presente que, como ya hemos señalado, el español es un mercado donde dichas empresas distribuyen ampliamente sus productos mediante numerosos puntos de venta. Por otra parte, hace ya una década que España reparó en el público italiano como objetivo de su oferta en turismo de salud y belleza, ${ }^{6}$ pues es bien conocida la preocupación del pueblo italiano por el culto al cuerpo ${ }^{7}$ así como el estatus socioeconómico medio-alto de este tipo de turista y su disponibilidad para viajar en cualquier época del año. La oferta española, en este sentido, es muy competitiva tanto en precios como en variedad y calidad de sus centros. Esta situación genera un mercado muy interesante de traducción inversa hacia el italiano.

El ámbito de las ferias internacionales también genera un abundante intercambio de información entre Italia y España. Así por ejemplo, desde hace más de cuarenta años, se celebra en Italia Cosmoprof, el evento internacional más importante en el sector de la cosmética profesional, plataforma internacional para el mundo del bienestar y la belleza, en el que participan cada año más de dos mil empresas procedentes de setenta países de todo el mundo, ${ }^{8}$ entre ellos España, que participó en 2013 con más de cien empresas. En este tipo de contexto, la traducción desempeña un papel fundamental, sobre todo en la fase previa de preparación y organización del evento, y, cómo no, también la interpretación, especialmente durante los días de celebración de la feria.

El carácter multidisciplinar de este bloque temático exige además del traductor conocimientos conceptuales y terminológicos de muchas otras ramas del saber - química, farmacología, bioquímica, dermatología, microbiología, anatomía y hasta historia del arte -, lo cual lo convierte en un ámbito que requiere una gran versatilidad y especialización por parte del estudiante de traducción.

Traducir textos de la industria cosmética implica también conocer aspectos legalesy tendencias de consumo de cada cultura. Es necesario conocer, por ejemplo, que en 2010 la normativa española sobre productos cosméticos se adaptó a la comunitaria: según el Reglamento (CE) no 1223/2009 del Parlamento Europeo y del Consejo, de 30 de noviembre de 2009, sobre los productos cosméticos, la

${ }^{6}$ Desde que en 2004 España participara por primera vez en Thermalia, la Feria de Turismo de Salud y Belleza, celebrada en la isla italiana de Ischia, la presencia de nuestro país en las principales ferias del turismo en Italia es constante, la última de las cuales ha tenido lugar recientemente en Rímini (TTG octubre 2013). Italia es el cuarto mercado emisor de turistas para España y el sexto para Andalucía.

${ }^{7} \mathrm{Su}$ consumo de productos de belleza y curas termales es de los más altos en Europa.

${ }^{8}$ En el enlace http://www.cosmoprof.it/la-fiera/la-fiera/ se pueden consultar los datos sobre sectores en que se divide la feria, participantes, visitantes, países de procedencia, etc. 
lengua en la que debe estar expresada determinada información (peso y volumen, fecha y lugar de embalaje, precauciones particulares de empleo, función del producto) será determinada por la legislación de los Estados miembros en los que el producto se ponga a disposición del usuario final. ${ }^{9}$

En cuanto a las tendencias de consumo, las modas y la forma de comercialización del producto, es bien sabido que, a pesar de la globalización actual, no en todos los países gustan los mismos colores en el mismo momento, ni se busca el mismo efecto en la piel o en el cabello, ni resulta igualmente llamativo un determinado modelo de belleza masculino o femenino. Recordemos en este sentido el ejemplo de M. C. Valdés Rodríguez (2004), sobre la publicidad de los tintes de pelo L'Oréal Casting en Reino Unido y en España: las diferencias afectaban a aspectos de lo más diversos, desde el nombre de la modelo (Rocío en España, Magali en Reino Unido) hasta el número de tonos disponibles en ambos países (16 en España, 14 en Reino Unido), pasando por la selección léxica y el tono elegido para las modelos fotografiadas ("cereza caoba rojizo", color de moda en España en aquella época, y "Casting Jamaica" en Reino Unido, tono de pelo muy oscuro y exótico para los británicos).

Una prueba de que hay conciencia sobre las particularidades de la traducción en este sector es la existencia de agencias especializadas en traducción de textos de cosmética, las cuales hacen hincapié en la importancia de elementos tales como creatividad, precisión, homogeneidad terminológica y conocimiento de la industria cosmética así como de la cultura y hábitos de consumo del destinatario de la versión traducida para llegar a un resultado exitoso. Algunas de estas empresas que incluyen el español y el italiano entre sus idiomas de trabajo son beautyterm LLC, ${ }^{10}$ Cosmetic Translation, ${ }^{11}$ Lipstick Translation. ${ }^{12}$

A su vez, desde el punto de vista temático, en este campo es posible encontrar subdivisiones distintas según el autor y el objetivo de la clasificación. Para nuestros fines, dividimos el sector de la cosmética en las siguientes grandes familias:

1) maquillaje

2) perfumería

3) productos para el aseo y cuidado del cuerpo/rostro

${ }^{9}$ A partir del 11 de julio de 2013, se derogó parcialmente el RD 1599/1997 de 17 de octubre (modificado por RD 2131/2004, de 29 de octubre, RD 209/2005, de 25 de febrero y RD 944/2010, de 23 de julio), sobre etiquetado, cuyo capítulo V art. 15 establece que la fecha de caducidad mínima, las precauciones particulares de empleo y la función del producto "deberán figurar al menos en la lengua española oficial del Estado”; en cambio la denominación del producto, la información sobre el fabricante (nombre o razón social, dirección o domicilio social) y la referencia de identificación, "podrán expresarse en las lenguas nacionales u oficiales de origen, cuando el producto proceda de países del territorio comunitario".

${ }^{10} \mathrm{http}: / /$ www.beautyterm.com/

$11 \mathrm{http}: / /$ www.cosmetictranslation.com

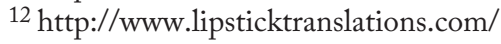


4) clínicas, centros de estética y spas

5) medicina estética

Cada uno de ellos es susceptible de ulteriores subdivisiones, como veremos más adelante en la categoría de productos para el cuidado del cuerpo y rostro, objeto temático del sitio web que constituirá nuestro encargo de traducción.

\section{RECURSOS DOCUMENTALES Y TERMINOLÓGICOS PARA LA} TRADUCCIÓN DE TEXTOS DE LA INDUSTRLA COSMÉTICA ${ }^{13}$

A pesar de la considerable demanda de recursos documentales y terminológicos para la traducción en este campo, las herramientas lexicográficas bilingües o multilingües en el sector de la cosmética que cuenten con el italiano y el español en su combinación lingüística no solo son escasas sino que datan de hace más de cuarenta años. Así por ejemplo, el Elsevier's lexicon of detergents, cosmetics and toiletries de G. Carrière (1966), vocabulario multilingüe que consta de dos partes, la primera, ${ }^{14}$ dedicada a los detergentes, incluye equivalentes en 19 idiomas, ${ }^{15} \mathrm{y}$ la segunda, dedicada a los cosméticos y productos de aseo, incluye equivalentes en inglés, francés, español, italiano, portugués, alemán, holandés y sueco. En 1978, el mismo autor realiza una revisión de esta obra publicando el Dictionary of surface active agents, cosmetics, and toiletries: English, French, German, Spanish, Italian, Dutch, Polish.

El panorama mejora ligeramente cuando se trata de lexicografía monolingüe: en español, encontramos obras como el Diccionario de ingredientes cosméticos de F. Carrasco (2005), ${ }^{16}$ que aclara las funciones de más de diez mil materias primas cuyas denominaciones -comunes para todos los Estados de la UEaparecen en el etiquetado de los productos cosméticos y resultan muchas veces incomprensibles para los consumidores. Además del diccionario en sí, esta obra ofrece información sumamente útil para quien desee iniciarse en el mundo de la cosmetología: funciones cosméticas principales, categorías de productos cosméticos, convenciones en la denominación de los ingredientes, etc. Completa la obra todo un capítulo referido a materia legislativa, tan importante en este ámbito, y sobre envasado y etiquetado de sustancias peligrosas.

Entre los años noventa y principios del siglo XXI, se publican otros diccionarios (Léxico de cosméticos y afeites en el Siglo de Oro, Diccionario breve

${ }^{13}$ Las referencias completas de todas las obras incluidas en este apartado se encuentran en la bibliografía final (v. Fuentes Lexicográficas).

${ }^{14}$ La primera parte es en realidad una edición revisada de la obra del mismo autor Detergents: a glossary of terms used in the detergents industry: in English, Norwegian, French, Russian [and Other Languages] (1960).

${ }^{15}$ Inglés, francés, español, italiano, portugués, alemán, holandés, sueco, danés, noruego, ruso, polaco, finés, checo, húngaro, rumano, griego, turco y japonés.

16 Se puede consultar un extracto en pdf en la siguiente dirección: http://www.imagenpersonal.com/di_in_in.htm 
de estética, La cosmética de la A a la Z, Diccionario de cosmetología, ${ }^{17}$ Diccionario cosmetológico de la esteticista) que, de forma más o menos extensa, incluyen entradas referidas a la estética y la cosmetología tanto en la actualidad como en épocas pretéritas. El grupo editorial Podium Ediciones, ${ }^{18}$ especializado en el mundo de la belleza, publica en red una revista mensual y varias obras de referencia: Quién es quién (Anuario de la Industria de la Perfumería y Cosmética en España), Diccionario de la Cosmética de Tratamiento, Diccionario de las Fragancias, Diccionario del Maquillaje, Diccionario de Tratamientos Capilares, $4^{\circ}$ Diccionario de la dermocosmética en Farmacia, que se actualizan cada año y cuentan con difusión nacional e internacional.

En italiano, contamos con el clásico Dizionario di Cosmetologia (1992) de G. Proserpio, ampliado, revisado y actualizado por E. Racchini en 2009. De estilo divulgativo, fue concebida como obra de consulta pero también de formación para expertos, proveedores, distribuidores y consumidores. Retrocediendo en el tiempo, encontramos los volúmenes Curiamoci con le erbe. Dizionario. Cosmesi e dietetica, publicados por Librex en 1971 y, más recientemente, la obrita Dizionario di bellezza: l'arte della cosmesi de M. S. De Salvia Baldini, publicada por Garzanti en 1995. Es este, a pesar de sus reducidas dimensiones, un diccionario especializado enciclopédico que aborda "el arte" de la cosmética desde un punto de vista diacrónico, incluyendo remedios y pócimas utilizadas en la Antigüedad así como técnicas de maquillaje y cirugía estética más modernas. Algunas entradas van acompañadas de ilustraciones (pinturas, esculturas, imágenes publicitarias), así como de información y anécdotas sobre el uso de los cosméticos a lo largo del tiempo, lo cual permite conocer la historia de la cosmética, su composición y beneficios.

Asimismo, se pueden encontrar glosarios -aunque no se presenten como talesen el interior de manuales dedicados a algún aspecto concreto de la cosmetología: son los denominados glosarios escondidos (Martínez de Sousa 2004). Así por ejemplo, el manual Dermocosmetologia. Dall'inestetismo al trattamento cosmetico de A. Bovero (2011) incluye en su segunda parte, dedicada a los cosméticos, más de cincuenta páginas con definiciones y explicaciones sobre las formas y los ingredientes cosméticos.

Pero es en Internet donde actualmente se encuentra el mayor número de recursos monolingües, bilingües y multilingües, ya se trate de glosarios, diccionarios o páginas informativas que incluyen frecuentemente breves glosarios monolingües o bilingües. ${ }^{19}$

17 En este diccionario al final de cada artículo se ofrecen los equivalentes en inglés.

18 Consultable en http://www.podiumediciones.es/productos/anuarios-y-diccionarios

19 Bases de datos orientadas al término como, por ejemplo, EURODICAUTOM, contienen registros del campo de la cosmética bajo la etiqueta de compuestos químicos $[\mathrm{COM}]$ o de área temática sin especificar. 
Presentamos a continuación una breve descripción de algunos de estos sitios de interés para las tareas de traducción entre el italiano y el español ${ }^{20}$ en el campo de la cosmética:

1) http://www.ccnphawaii.com/glossary.it.htm

Glosario para el Fabricante Moderno de Jabón / Glossario del Saponaio Moderno. Incluye términos, definiciones y abreviaturas sobre la fabricación del jabón. El lemario original está en inglés y según la LT que el usuario elija - francés, alemán, español e italiano -, el idioma del equivalente y de la definición va cambiando. Si se elige inglés, tanto el lema como la definición aparecen en inglés. En realidad las definiciones en los restantes idiomas son traducciones de las originales en inglés. No permite búsquedas por término, solo alfabéticas.

2) http://www.sitiosespana.com/diccionarios/COSMETICA/

Diccionario de estética y cosmética. Monolingüe en español. Incluye definiciones de términos, locuciones, siglas y extranjerismos, así como remisiones internas entre lemas. No permite búsquedas por término, solo alfabéticas.

3) http://estetica.index.es/subpaginas.php?a=150

Diccionario Estético de Cosmética, Bienestar y Belleza. Monolingüe en español. Lemario reducido que incluye siglas, locuciones y extranjerismos. No permite búsquedas por término, solo alfabéticas.

4) http://acacia.pntic.mec.es/sazg0000/glosario2009.htm

Glosario de términos, principios activos, correctivos y excipientes utilizados en las elaboraciones. Realizado por el profesor Azuara Gascón. ${ }^{21}$ Monolingüe en español. Su amplio lemario (más de 1200 entradas) incluye nombres comerciales, siglas y extranjerismos. Remisiones internas entre lemas. No permite búsquedas por término, solo alfabéticas.

5) http://www.ecoaldea.com/old/preparaciones/preparados.htm

Página informativa en español con más de 30 categorías de preparados en fitoterapia.

6) http://www.mundobelleza.com/glosario/glosario.htm

Monolingüe en español. Lemario que incluye, entre otros términos, nombres de tratamientos estéticos, enfermedades de la piel y la circulación, siglas, nombres de compuestos orgánicos y químicos en latín, castellano e inglés.

7) http://dimetilsulfuro.es/2013/08/08/cosmetica-para-todos-los-publicos/ Artículo que ayuda a comprender el significado de los principales ingredientes cosméticos utilizando la nomenclatura INCI. No permite búsquedas.

20 Restringiremos la búsqueda a los recursos que utilizan español peninsular.

${ }^{21}$ Catedrático del Dpto. de Imagen Personal, I.E.S. Almirante Bastarreche (Cartagena, Murcia). 
8) http://www.beautymarket.es/glosario_cosmetologia.php

Glosario con entradas muy completas que incluye términos del campo de la dermatología, el cabello, los perfumes y la piel. Incluye nombres científicos, siglas y locuciones. No permite búsquedas.

9) http://es.scribd.com/doc/96697429/GLOSARIO-DE-COSMETOLOGIAY-ESTETICA

Glosario monolingüe en español, extenso y completo pues incluye siglas, fraseología, nombres científicos y extranjerismos, así como términos procedentes de otros campos (gameto, hercio, impedancia...). No permite búsquedas.

10) http://popestetica.com/glosario/

Glosario monolingüe en español. Incluye fraseología, nombres científicos, variantes gráficas, extranjerismos, siglas, remisiones internas entre términos. No permite búsquedas.

11) http://www.guioteca.com/belleza/glosario-de-estetica-lo-que-debes-saber/ Glosario monolingüe en español. Pocos lemas y referidos en exclusiva a los tratamientos estéticos.

12) http://www.vogue.es/belleza/articulos/cosmetica-de-la-a-a-la-z/5097 Glosario monolingüe en español incluido en el portal de la revista Vogue. Incluye veinte términos con sus correspondientes fotografías, referidos a la piel y a tratamientos antienvejecimiento.

13) http://diccionarios.hola.com/belleza/indice/a/

Glosario monolingüe en español incluido en el portal de la revista Hola. Incluye siglas, extranjerismos, locuciones. Permite búsquedas con un buscador específico.

14) http://www.monografias.com/trabajos26/medicamentos-vegetal/ medicamentos-vegetal.shtml

Glosario monolingüe en español sobre preparaciones en fitoterapia. Remisiones internas a otros términos en forma de hipervínculos.

En cuanto a los recursos online en lengua italiana, destacaremos los siguientes:

1) http://www.unipro.org/home/it/

Sitio web de la Asociación Italiana de Empresas Cosméticas.

2) http://www.maldyitaliana.it/resources/pdf/pdc.pdf

Piccolo dizionario delle materie prime cosmetiche. Breve diccionario monolingüe en italiano sobre ingredientes cosméticos. ${ }^{22}$ Fuente desconocida.

22 Muchos diccionarios en red, incluidos en blogs, páginas sobre cosmética, etc. son en realidad copias exactas de este diccionario. 
3) http://www.abspace.it/WellnessSpace/glossario_Cosmetici. asp?key=Cosmetici\&page $=1$

Incluye 285 voces, en algunas de las cuales, más que una definición, se ofrece información sobre su función o composición. Si se pincha sobre el lema, realiza una búsqueda de dicho término en google.

4) http://www.biodizionario.it/

Guía para el consumo responsable de cosméticos, realizada por Fabrizio Zago, químico industrial y asesor de Ecolabel. Ofrece un listado de ingredientes habituales en los cosméticos con una serie de círculos verdes, amarillos y rojos, según el uso recomendado o no de los mismos. No ofrece definiciones sino que informa sobre la naturaleza del ingrediente en cuestión.

5)http://www.newestetica.it/joomla2/index.php?option=com_content\&view $=$ article $\&$ id $=57 \&$ Itemid $=63$

Il dizionario del make-up es un breve glosario incluido en la página del Istituto New Estetica Giuliana donde se proporcionan principalmente explicaciones sobre los productos de maquillaje de la casa, muchas de ellas útiles de todas formas para el público en general, no solo para los clientes de la empresa.

6) http://www.ilmiomakeup.it/dizionario-cosmetico-termini/

Se trata de un diccionario muy breve, formado en su mayor parte por extranjerismos, muy utilizados en el lenguaje de la cosmética en italiano.

7) http://www.abc-cosmetici.it/index.php/category/conoscere-i-cosmetici/ glossario/a/

El sitio web http://www.abc-cosmetici.it es un portal recomendado por Unipro para conocer mejor los productos cosméticos y sus ingredientes. En el enlace que señalamos aquí encontramos un glosario monolingüe que incluye siglas, extranjerismos, nombres propios, nombres de leyes y remisiones internas entre voces.

8) http://www.donna-in.com/2011/07/dizionario-del-make-up-il-trucco-dallaa-alla-z/

Breve diccionario monolingüe sobre productos y técnicas de maquillaje.

9) http://www.lifegate.it/persone/stile-di-vita/

breve_dizionario_degli_ingredienti_cosmetici_nocivi

Breve diccionario con aquellos ingredientes cosméticos que pueden resultar perjudiciales para determinados tipos de pieles. Aparecen divididos en categorías: aditivos, emulsionantes, conservantes y tensioactivos. 


\section{LINGÜÍSTICA DE CORPUS YTRADUCCIÓN}

Partimos de la definición de corpus proporcionada por Alvar Ezquerra, Blanco Rodríguez y Pérez Lagos (1994: 9-10):

Un corpus es un conjunto homogéneo de documentos lingüísticos de cualquier tipo (orales, escritos, literarios, coloquiales, etc.) que se toman como modelo de un estado o nivel de lengua predeterminado, al cual representan o se pretende que representen.

En cuanto a los tipos de corpus existentes, se han establecido tipologías diferentes según el criterio de clasificación adoptado (Corpas 2001: 157-159):23

1) Según la distribución de los textos en el corpus, estos pueden ser: grande o extenso, equilibrado, piramidal, monitor, paralelo, comparable;

2) Según la especificidad de los documentos que contienen: general, especializado, genérico, canónico, periódico o cronológico, diacrónico;

3) Según la cantidad de texto recogido en los documentos que lo componen: corpus textual, de referencia, léxico;

4) Según la codificación y anotación del corpus: anotado y no anotado;

5) Según la documentación que acompaña a los textos: documentado, no documentado.

Los corpus paralelos y comparables son los más utilizados en los estudios de traducción. Los primeros, que pueden ser bilingües o multilingües, incluyen textos originales junto con sus correspondientes traducciones a una o más lenguas, respectivamente. Los comparables, por su parte, incluyen textos similares originales en dos o más lenguas (por lo que pueden ser asimismo bilingües o multilingües). Se habla, por último, de corpus comparable monolingüe para denominar aquel corpus formado por textos originales y traducidos en una misma lengua.

Son muchas las disciplinas que deben a la lingüística de corpus importantes aportaciones: la lexicografía, la didáctica de segundas lenguas, la terminología, la sociolingüística y, cómo no, la traducción e interpretación. En este último caso, los corpus se han utilizado con objetivos muy diversos: la comparación interlingüística, el análisis de las características de una determinada lengua traducida en comparación con el empleo de esa misma lengua en textos originales, ${ }^{24}$ el desarrollo de sistemas de traducción automática y herramientas

${ }^{23}$ Esta autora se basa a su vez en las propuestas de Torruella y Llisterri (1999), Baker (1995) y Johansson (1998).

${ }^{24}$ Para un estudio del italiano de las traducciones comparado con el italiano de las producciones espontáneas, véanse Cardinaletti y Garzone (2005) y Cardinaletti (2012). 
$\mathrm{TAO}^{25}$ y la formación de traductores e intérpretes, como vamos a ver a continuación.

En efecto, a principios del siglo XXI comienzan a proliferar los estudios dedicados al corpus como elemento curricular tanto en la enseñanza de lenguas extranjeras como en la formación de traductores e intérpretes: Aston (2000), Bernardini y Zanettin (2000), ${ }^{26}$ Zanettin (2001), Kenny (2001), Bowker y Pearson (2002), Maia (2003), Corpas Pastor (2001, 2002, 2004a, 2004b), Zanettin, Bernardini y Stewart (2003), Rodríguez Inés (2008), entre otros.

En nuestro caso, para las clases de traducción especializada, nos interesará enseñar a los alumnos a crear y a utilizar eficazmente una herramienta que les puede resultar sumamente útil además de relativamente fácil de construir: un corpus $a d$ hoc que les ayude a hacer frente a un encargo de traducción italiano/ español de un sitio web de una casa italiana de cosméticos.

\section{CREACIÓN DE UN CORPUS AD HOC}

Define Corpas Pastor (2004a: 236) el corpus ad hoc de la siguiente manera:

Un corpus ad hoc es un corpus virtual que se compila puntualmente para la realización de un determinado encargo de traducción en cualquier dirección (directa, inversa o indirecta).

Es obligado, pues, en primer lugar, exponer el encargo de traducción que propusimos a los alumnos. Se trataría de una simulación de encargo real, si bien, en casos como este, sería perfectamente posible proponer a la empresa la publicación de la traducción española, algo que siempre aconsejamos a los alumnos, pues de iniciativas como esta pueden surgir relaciones profesionales inesperadas y, por qué no, duraderas.

Vamos a traducir al español la página web de la empresa Rebis Cosmetici Naturali Made in Italy (http://www.rebis-cosmetici.it/web/), 27 que se dedica a la fabricación de cosméticos formulados por el médico y herborista Roberto Mola utilizando sustancias naturales procedentes, según información de la propia página web, de la ladera volcánica del Monte Amiata (Toscana) y de países lejanos como Méjico, China o Perú. Vamos a suponer que esta empresa pretende ampliar su mercado dándose a conocer entre el público español (no hispanoamericano), por lo que quiere publicar sus contenidos en castellano. En

\footnotetext{
25 Traducción asistida por ordenador (en inglés CAT: Computer Assisted Translation).

${ }^{26}$ Profesores en la SSLMIT (Scuola Superiore di Lingue Moderne per Interpreti e Traduttori) de Forlì (Universidad de Bolonia), actualmente denominada Sede di Forlì della Scuola di Lingue e Letterature, Traduzione e Interpretazione, y principal centro de investigación sobre el uso del corpus en la didáctica de la traducción.

${ }^{27}$ La sede de esta empresa se encuentra en Cremona (Lombardía). Sus productos se venden en herboristerías, tiendas ecológicas y a través del catálogo Panda Shop a los socios del WWF.
} 
la realidad lo más probable es que pidieran traducir el sitio web completo, ${ }^{28}$ pero este encargo, por razones prácticas, lo limitaremos a tres de sus líneas de productos: la línea capilar, la línea corporal y la línea de productos para la boca, barba, nariz y oídos.

El sitio web de Rebis presenta una estructura clara que permite una fácil navegación y un diseño muy atractivo con dibujos y caracteres infantiles en tonos pastel que evocan la inocencia, lo natural, saludable y artesano. Los envases y etiquetas de los productos presentan la misma apariencia. En cuanto a la marca, recordemos que el nombre Rebis aparece en textos de alquimistas y representa a un ser mitológico, humano pero hermafrodita, que simboliza la dualidad, la perfección y lo inalcanzable (gráficamente se representa como un varón y una fémina fundidos en un solo cuerpo). Encontramos esta imagen en el sitio web, en forma de un rey y una reina unidos en un solo ser, junto con otros temas propios de la alquimia del siglo XVI (la rosa roja y los frascos donde se preparan pócimas mágicas) "che invitano ad un uso concreto ed insieme simbolico dei cosmetici naturali della Rebis".

El encargo de traducción no incluye el tratamiento de las fotografías que acompañan al texto y que representan los envases con los nombres del producto en italiano, pues por el momento la empresa no desearía invertir en cambiar el texto de dichos envases. ${ }^{29}$ Sí serían objeto de traducción, en cambio, las fichas técnicas que completan la información sobre cada producto, a las cuales se accede mediante un hipervínculo, y que reproducen, entre otras informaciones, el texto impreso en el envase correspondiente.

En cuanto al género del TO, podríamos considerarlo un híbrido entre diversas clases de texto: estamos ante un sitio web ${ }^{30}$ que presenta material informativo y al mismo tiempo publicitario sobre la casa Rebis y los productos que comercializan. En concreto, la sección "I prodotti", parte de la cual constituye nuestro encargo de traducción, es de hecho un catálogo en línea con la descripción de las propiedades, composición y usos de cada uno de los productos, junto con la reproducción del texto de las etiquetas de los envases. Coexisten, pues, en el TO las funciones informativa e instructiva (con opción, propia de los textos publicitarios). En cuanto al grado de especialización, tengamos en cuenta que el emisor es un especialista (los textos han sido redactados por el propio laboratorio

28 Aunque no faltan sitios web que deciden traducir solo parcialmente su contenido, dejando en la LO aquella información considerada menos importante o más vinculada a la cultura de origen (esta es la diferencia entre la simple traducción y la localización de sitios web).

${ }^{29}$ Esta política de traducir página web pero no los productos que llegan a las tiendas españolas la ponen en prácticas otras empresas italianas como, por ejemplo, Bottega Verde (www. bottegaverde.es) o Deborah Milano (http://www.deborahmilano.es).

30 Desde el punto de vista técnico, traducir un sitio web no debería representar un problema para los alumnos, pues en el mismo curso y semestre están aprendiendo a utilizar herramientas como WebBudget, OmegaT, Google Translator Toolkit o Trados en la asignatura Traducción de software y páginas web C (Italiano). 
fabricante) y que el receptor puede ser un especialista (químico interesado en la composición de los cosméticos), un semi-especialista (distribuidor de productos cosméticos, por ejemplo) hasta un potencial cliente completamente lego en la materia, por lo que podemos afirmar que nos encontramos ante un texto propio de la comunicación semi-especializada.

Antes de comenzar la fase de búsqueda de la información que formará parte del corpus en español, deberemos establecer unos criterios de diseño básicos ${ }^{31}$ que nos servirán de guía para elegir o descartar documentos:

1) propósito de la compilación: recopilar textos en la LT (español) que ayuden al alumno a abordar con éxito el encargo de traducción y pueda ser utilizado en la labor de documentación, para un mejor análisis y comprensión del TO y, por último, en la producción del TT;

2) tamaño: al ser un corpus especializado pensado para un encargo de traducción concreto, no será posible ni necesario compilar un corpus muy extenso;

3) medio: escrito, aunque al tratarse de un corpus virtual, los textos serán digitales, no impresos ni escaneados, sino procedentes todos de Internet;

4) temática: como hemos señalado anteriormente, son tres las líneas de cosméticos que nos interesan para el encargo: productos para el cabello, para el conjunto formado por boca, barba, nariz y oídos, y cosmética para el cuerpo; asimismo, para la fase de documentación, serán igualmente interesantes aquellos documentos que describan las propiedades de los ingredientes que entran en la composición de los productos;

5) tipo y género textual: en principio descargaremos aquellos textos que correspondan en forma y función al TO, es decir, material informativo/ publicitario en red, si bien, como veremos, es posible que debamos subdividir el corpus en secciones según los géneros textuales que nos puedan ser de utilidad; 6) autoría: para conseguir el mayor grado de fiabilidad posible dentro de este campo, intentaremos recoger textos procedentes de empresas de cosmética, farmacias, parafarmacias, siempre a ser posible españolas para que el material no sea en realidad una traducción de otra lengua; asimismo pueden resultar de interés aquellos blogs escritos por especialistas en el dominio que nos interesa; 7) fecha: buscaremos textos actuales, entendiendo por tales los publicados en los últimos cinco años; en las páginas web de donde extraeremos la información deberá aparecer la fecha de la última actualización, lo cual nos dará una pista sobre la actualidad de los contenidos y sobre la atención que recibe dicha página por parte de quienes la crearon;

8) lenguas: nos ceñiremos a los documentos redactados en español peninsular.

De entre los distintos tipos posibles de corpus, ya comentados en el epígrafe anterior, optamos por compilar un corpus virtual, monolingüe y especializado, donde reuniremos textos comparables, del mismo tipo y género textual, la

31 Criterios elaborados por Bowker y Pearson (2002: 54) apud Castillo Rodríguez (2009). 
misma temática y mismo nivel de especialización. ${ }^{32}$ La siguiente tabla resume las características principales de nuestro corpus:

\begin{tabular}{|l|l|l|}
\hline Tipo de corpus: & Virtual, comparable, $a d$ hoc, monolingüe, especializado & \\
\hline Género textual: & Página web (ficha informativa/técnica) & \\
\hline Tema: & Línea capilar & 57.585 pal. \\
\hline & Línea boca, barba, nariz, oídos & $34.827 \mathrm{pal}$. \\
\hline & Línea corporal & $33.567 \mathrm{pal}$. \\
\hline Dimensión total & & 125.979 pal. \\
\hline
\end{tabular}

Para la creación de dicho corpus seguiremos el protocolo propuesto por G. Corpas (2001, 2004a, 2004b). Procedemos, pues, a ilustrar el método para compilar y explotar este corpus específico para el encargo de traducción asignado.

\subsection{Análisis del TO e identificación de palabras clave}

En primer lugar, convertiremos el formato. $\mathrm{html}$ del texto que hemos de traducir a formato .txt, para poder manejar el TO con la herramienta de gestión de corpus Wordsmith (WS). El recuento de palabras del TO nos indica que consta de 7.600 palabras, si bien el porcentaje de repetición es alto. ${ }^{33}$

Puesto que el TO comprende tres líneas de productos muy distintas entre sí, consideramos más eficaz subdividir nuestro corpus, que llamaremos CCR (Corpus Cosmética Rebis) en tres subcorpus, correspondientes a cada una de las líneas: $\mathrm{CCa}$ (Corpus Cabello), CBBNO (Corpus Boca Barba Nariz Oídos) y $\mathrm{CCu}$ (Corpus Cuerpo).

Comenzaremos con la línea capilar, a la que, por razones de espacio, restringiremos el presente estudio. Identificaremos en primer lugar las palabras clave del TO (linea per capelli) mediante un análisis estadístico de frecuencias que nos permite realizar WS. Este análisis muestra un total de 5532 ocurrencias (tokens) a partir de 757 palabras-tipo (types), lo cual indica una riqueza léxico del 13,77\%. La siguiente tabla nos muestra las 50 palabras más frecuentes en este primer subcorpus (línea capilar), una vez eliminadas las palabras gramaticales más frecuentes mediante la denominada stop list:

\begin{tabular}{|l|l|l|l|}
\hline $\mathbf{N}$ & Word & Freq. & $\mathbf{\%}$ \\
\hline Lemmas & Set & & \\
\hline $\mathbf{1}$ & CAPELLI & 90 & 1.63 \\
\hline $\mathbf{2}$ & PROPOLI & 57 & 1.03 \\
\hline $\mathbf{3}$ & SHAMPOO & 54 & 0.98 \\
\hline $\mathbf{4}$ & OIL & 51 & 0.92 \\
\hline
\end{tabular}

${ }^{32}$ Son los que tradicionalmente se conocen en clase como textos paralelos.

33 Por ejemplo, el último párrafo del texto en los envases es el mismo en todos los casos. Dicho texto, además, contiene gran parte de la información ofrecida en la descripción general del producto fuera de la ficha técnica. 


\begin{tabular}{|l|l|l|l|}
\hline $\mathbf{5}$ & OLI & 49 & 0.89 \\
\hline $\mathbf{6}$ & ESSENZIALI & 48 & 0.87 \\
\hline $\mathbf{7}$ & OLIO & 46 & 0.83 \\
\hline $\mathbf{8}$ & API & 44 & 0.80 \\
\hline $\mathbf{9}$ & REBIS & 43 & 0.78 \\
\hline $\mathbf{1 0}$ & LAURYL & 38 & 0.69 \\
\hline $\mathbf{1 1}$ & \# & 36 & 0.65 \\
\hline $\mathbf{1 2}$ & NATURALE & 36 & 0.65 \\
\hline $\mathbf{1 3}$ & COLLINARE & 35 & 0.63 \\
\hline $\mathbf{1 4}$ & SOSTANZE & 35 & 0.63 \\
\hline $\mathbf{1 5}$ & TOSCANA & 33 & 0.60 \\
\hline $\mathbf{1 6}$ & ASTUCCIO & 30 & 0.54 \\
\hline $\mathbf{1 7}$ & AZIONE & 30 & 0.54 \\
\hline $\mathbf{1 8}$ & CARTA & 30 & 0.54 \\
\hline $\mathbf{1 9}$ & DERIVATI & 30 & 0.54 \\
\hline $\mathbf{2 0}$ & ECOLOGICA & 30 & 0.54 \\
\hline $\mathbf{2 1}$ & COCCO & 28 & 0.51 \\
\hline $\mathbf{2 2}$ & INGREDIENTI & 27 & 0.49 \\
\hline $\mathbf{2 3}$ & LAVANDA & 25 & 0.45 \\
\hline $\mathbf{2 4}$ & ESTRATTI & 24 & 0.43 \\
\hline $\mathbf{2 5}$ & YLANG & 24 & 0.43 \\
\hline $\mathbf{2 6}$ & ALCOHOL & 23 & 0.42 \\
\hline $\mathbf{2 7}$ & KASHMIR & 23 & 0.42 \\
\hline $\mathbf{2 8}$ & ESTRATTO & 22 & 0.40 \\
\hline $\mathbf{2 9}$ & ACIDO & 21 & 0.38 \\
\hline $\mathbf{3 0}$ & MAIS & 21 & 0.38 \\
\hline $\mathbf{3 1}$ & ED & 20 & 0.36 \\
\hline $\mathbf{3 2}$ & PRODOTTI & 20 & 0.36 \\
\hline $\mathbf{3 3}$ & SULFATE & 20 & 0.36 \\
\hline $\mathbf{3 4}$ & AMMONIUM & 19 & 0.34 \\
\hline $\mathbf{3 5}$ & PIÜ & 19 & 0.34 \\
\hline $\mathbf{3 6}$ & ALCOOL & 18 & 0.33 \\
\hline $\mathbf{3 7}$ & COCOAMPHODIACETATE & 18 & 0.33 \\
\hline $\mathbf{3 8}$ & DISODIUM & 18 & 0.33 \\
\hline $\mathbf{3 9}$ & FUNZIONE & 18 & 0.33 \\
\hline $\mathbf{4 0}$ & GLUCOSIDE & 18 & 0.33 \\
\hline $\mathbf{4 1}$ & INGREDIENTS & 18 & 0.33 \\
\hline $\mathbf{4 2}$ & LOZIONE & 18 & 0.33 \\
\hline $\mathbf{4 3}$ & ML & 18 & 0.33 \\
\hline $\mathbf{4 4}$ & NATURALI & 18 & 0.33 \\
\hline $\mathbf{4 5}$ & PRESENTE & 18 & 0.33 \\
\hline $\mathbf{4 6}$ & RIPRODUZIONE & 18 & 0.33 \\
\hline $\mathbf{4 7}$ & SCHEDA & 18 & 0.33 \\
\hline $\mathbf{4 8}$ & SOLO & 18 & 0.33 \\
\hline $\mathbf{4 9}$ & STAMPATO & 18 & 0.33 \\
\hline $\mathbf{5 0}$ & TECNICA & 18 & 0.33 \\
\hline & & & \\
\hline
\end{tabular}


Esta tabla nos permite hacernos una idea de la red conceptual y terminológica del TO: se nos presenta una línea de champús (shampoo) específicos elaborados a base de ingredientes y extractos naturales (ingredienti e estratti naturali) como propóleo (propoli), coco (cocco), maíz (mais) y diversos aceites esenciales (oli essenziali). Algunos de estos ingredientes provienen de las colinas toscanas (collinare toscana). Ciertos productos de esta línea tendrán como ingrediente principal un extracto de la lana de cachemira (kashmir), mientras que otros como la lavanda (lavanda) y el ylang-ylang (ylang-ylang) parecen repetirse en varias líneas, vista la frecuencia de aparición de estos términos. Por esta misma razón, en la ficha técnica que acompaña a cada tipo de champú deberán aparecer sustancias como ácido (acido), sulfato (sulfate), amonio (ammonium), alcohol (alcool), disódico cocoanfodiacetato (disodium cocoamphodiacetate) y glucósido (glucoside).

\subsection{Localización de documentos y almacenamiento de datos}

Tras el análisis de las palabras más frecuentes en el TO, utilizaremos los equivalentes en español de dichas palabras clave para realizar búsquedas en la red de los documentos que pasarán a formar parte del subcorpus CCa. Recurriremos a la opción de búsqueda avanzada en Google para seleccionar idioma español, páginas procedentes únicamente de España y aplicar técnicas de búsqueda booleana que nos permitan acceder a textos especializados con la menor cantidad de "ruido" 34 posible. Las palabras clave serán champú, ingredientes, extractos naturales, aceites esenciales, propóleo.

Los resultados de esta búsqueda nos han permitido compilar un subcorpus de 57.585 palabras, subdividido a su vez en dos secciones según el género textual de los documentos que las componen:

A) La primera sección, que hemos denominado CcaCat, contiene catálogos en línea incluidos en las páginas web de farmacias, parafarmacias, tiendas especializadas y empresas fabricantes de cosmética, en los que se describen las propiedades, composición y función de diferentes productos para el cuidado del cabello. Hemos excluido toda la información referente a otro tipo de productos, como perfumes, protección solar, maquillaje, cosmética para bebés, etc. Esta sección la componen 25.379 palabras.

B) La segunda sección, que hemos denominado CcaBlog, contiene información procedente de blogs y reportajes creados por especialistas en la materia: farmacéuticos, homeópatas, acupuntores, naturópatas, masajistas, etc. Hemos incluido estos textos por proporcionar información muy útil

34 Con "ruido" nos referimos a documentos que no son pertinentes para nuestro corpus pero que aparecen entre los resultados de nuestra búsqueda. 
sobre los ingredientes cosméticos, la cual puede ayudar mucho en la fase de documentación. ${ }^{35}$ Esta sección la componen 32.206 palabras.

Todos los documentos que pasarán a formar parte del corpus se encuentran originalmente en formato html, por lo que será preciso convertirlos a texto plano (.txt), que es, como ya hemos señalado, el único que manejan los programas de concordancias como WS. Además, iremos registrando en una tabla Excel las referencias de los documentos que componen nuestro corpus, indicando la siguiente información: código de registro (formado por números consecutivos desde el 1, las siglas de la sección, sigla del idioma), título de la página web, dominio (farmacia, cosmética natural, parafarmacia, terapias, etc.), género (catálogo, blog, reportaje), tipo textual (informativo, argumentativo, etc.), URL, categoría/subcategoría (cuidado del cabello, remedios caseros, línea corporal, tratamientos, etc.). ${ }^{36}$

\subsection{Explotación del corpus $C C R$}

Como ya indicamos anteriormente, el corpus compilado será de utilidad en las diversas fases del proceso de traducción: análisis del TO, labor de documentación y producción del TT.

En el apartado 5.1 nos hemos dedicado al análisis del TO, por lo que ahora ilustraremos cómo utilizar el corpus en las otras dos etapas mediante ejemplos extraídos del subcorpus CCa.

Identificaremos en primer lugar algunos problemas terminológicos que podrán ser resueltos gracias a la consulta del corpus.

\section{1) propoli d'api}

Este ingrediente forma parte de todos los champús de la Rebis, por lo que es fundamental comprender bien a qué sustancia se refiere y utilizar la denominación correcta en español.

En el programa WS, utilizamos la aplicación de concordancias Concord, la cual permite obtener listados de aparición de una determinada palabra rodeada de un cotexto mayor o menor en función de las preferencias del usuario. Indicamos al programa que ha de buscar en los archivos correspondientes a ambas secciones del subcorpus (pestaña Texts $>$ Choose Texts now) y a continuación especificamos el término o secuencia para los que deseamos obtener las concordancias. Partiremos del nodo $p r ? p$ ? $l^{*}$, donde el comodín ? sustituye cualquier carácter o signo de puntuación $\mathrm{y}^{*}$ sustituye un número indeterminado de letras: ${ }^{37}$

35 Evitamos los blogs redactados por usuarios de los cosméticos, pues sus textos suelen ser divulgativos y poco especializados, además de poco fiables en lo que a redacción y ortografía se refiere.

${ }^{36}$ Cf. Castillo Rodríguez (2009) para la compilación de un corpus ad hoc paralelo multilingüe.

${ }^{37}$ Hemos usado el comodín ? para incluir tanto las versiones con tilde como sin ella. 
Esta búsqueda nos proporciona unos resultados que van a sernos muy útiles tanto para la comprensión del concepto como para la búsqueda del equivalente en español, pues encontramos varias definiciones del ingrediente, así como explicaciones sobre su composición y propiedades:

El Propóleo es un conjunto de resinas, gomas y polen que recogen las abejas de las plantas y árboles, las abejas mezclan estas sustancias con sus propias secreciones para reparar y proteger la colmena.

El propóleo o própolis es una preciosa materia que las abejas producen en pequeña cantidad para la protección de sus colmenas. Estas lo obtinen recolectando sustancias resinosas de determinadas plantas y brotes, que modifican con las enzimas de su saliva.

Esto explica que el Propóleo tenga grandes propiedades regenerativas, acción antibiótica, antivírica, antifúngica, antiséptica y cicatrizante.

Entre los 50 resultados obtenidos, descartamos 10, referidos a la línea de productos Propoline, al champú Propol-mel, a los compuestos propylene glycol I propilenglicol (glicol de propileno) y propylparaben y la palabra propulsores, recuperados por cumplir los requisitos de la búsqueda realizada.

Entre las 40 ocurrencias restantes, 25 corresponden a propóleo (con o sin tilde) y 15 a própolis. Curiosamente, no se da ninguna ocurrencia de propóleos, que es la forma recogida por el DRAE. Entre las 25 ocurrencias de propóleo, en 8 ocasiones la palabra no lleva tilde. La clara inferioridad en número y el hecho de que en todas las ocasiones dicha grafía procede del corpus basado en blogs, nos hace descartar esta posibilidad, pues se trata obviamente de un error ortográfico.

Los resultados arrojados por la consulta del corpus nos indican, por tanto, que el equivalente buscado es propóleo, actuando como posible sinónimos própolis (ambos se presentan explícitamente como sinónimos en 3 ocurrencias):

El propóleo o própolis es una sustancia que obtienen las abejas de las yemas de los árboles y que luego procesan convirtiéndola en un potente antibiótico con el que protegen la colmena de bacterias y hongos.

Cuando utilizamos el término Propóleo o Própolis nos referimos a una serie de sustancias balsámicas, de consistencia gomosa, resinosa y viscosa y de un color amarillo pardo, que las abejas obtienen de las yemas y de los exudados de algunas especies de árboles de hoja perenne (abeto, abedul, haya, fresno, alerce, olmo, aliso, castaño de indias, pino, álamo, roble, etc.). Después de recolectar estas substancias, las abejas las reelaboran incorporando cera.

El propóleo o própolis es una preciosa materia que las abejas producen en pequeña cantidad para la protección de sus colmenas.

\section{2) balsamo attivante}

Por la ficha técnica que acompaña a este producto en el TO, sabemos que en su composición entran el ginseng coreano y el propóleo, que realiza acción 
preventiva contra la caída del cabello, a la vez que restaura la película lipídica que protege los cabellos, tonificándolos y devolviéndoles su vigor natural para que se vuelvan más luminosos y fáciles de peinar.

Para comprobar qué adjetivo acompaña en español al tipo de producto suavizante que consigue estos objetivos, vamos a buscar las ocurrencias de acondicionador en nuestro corpus, en esta ocasión especificando que en su contexto próximo aparezca la palabra caída. Para ello, realizaremos una búsqueda avanzada de palabra en contexto, denominada context word(s) en WS. Como palabra base de la búsqueda, seleccionamos acondicionador y buscamos contextos que incluyan caída entre las 10 posiciones a la izquierda (L10) y a la derecha (R10). Los contextos que no incluyan dicha palabra no serán, por tanto, tenidos en cuenta. Con estos criterios, la consulta del corpus no ofrece ningún resultado, por lo que deducimos que los catálogos de productos que hemos tomado como base para el corpus no incluyen acondicionadores anticaída. Realizamos, pues, la misma consulta con las palabras base champú y sérum.

Para ello, utilizamos la secuencia "champ?/s?rum" en la búsqueda simple 'Search Word' y “*ca?da” en 'Context Word(s)\&Context Search Horizons' especificando (L10) y (R10):38

Los adjetivos que acompañan a champú y a sérum en las líneas de concordancias obtenidas son los siguientes: tratante, anticaída, equilibrante, anticaída tonificante, nutri-reparador, concentrado anticaida, eficaz, fortificante:

Para optimizar la eficacia del tratamiento anticaída, se aconseja utilizar en complemento el champú tratante.

Champú equilibrante $200 \mathrm{ml}$ - EARTH LINE

Champú anticaida tonificante Apivita

Champú anticaida tonificante con lupino y romero

Tratamiento contra la caída del cabello prematura. Es un producto VICHY DERCOS - DERCOS Champú Nutri-Reparador

CHAMPÚ CONCENTRADO ANTICAÍDA + ANTICASPA. -Rhatma Therapy -

Champú eficaz contra la caída del cabello

Serum anticaída + antirrotura

Serum Fortificante Anticaída

Recordemos lo que decía el TO sobre las propiedades del balsamo attivante:

In forza di questi effetti concatenati il Balsamo Attivante si oppone alle più frequenti condizioni che favoriscono la caduta eccessiva dei capelli

${ }^{38}$ Los signos de interrogación prevén las grafías de champú y sérum sin tilde. 
Accediendo al texto completo correspondiente a las ocurrencias de tonificante y fortificante, ${ }^{39}$ comprobamos que son efectivamente adjetivos relacionados con la lucha contra la caída del cabello.

Champú anticaida tonificante Apivita

Ideal para los casos de pérdida de cabello estacional, especialmente durante la primavera y el otoño. Ayuda a tu cabello con un tratamiento natural, sin productos químicos, en estas etapas de pérdida de mucho cabello. Apivita reemplaza el agua con infusión de romero para ofrecer antioxidantes, tonificantes, y una acción antiséptica y anti-irritante.

Champú anticaida tonificante con lupino y romero para cabello débil y evitar su caída. Fortalece y nutre el pelo. Revitaliza el folículo del pelo, con altramuz, romero, ginseng, cola de caballo, vitaminas, oligoelementos y miel.

Serum Fortificante Anticaída con complejo activo quinina-cafeína, caída reaccional, cabello desvitalizado.

Asociación exclusiva de quinina y cafeína.

La Quinina, activo con poderosas propiedades fortificantes, está concentrada en un extracto del que hemos demostrado y patentado las propiedades estimulantes en el bulbo piloso. Vehiculizada por la cafeína, molécula con propiedades anticaída demostradas, la eficacia de la quinina queda sobreactivada.

Parece ser que tanto la capacidad de tonificar como de fortificar parecen caracterizar a productos que frenan la caída del cabello. Por tanto, para decidir entre tonificante y fortificante, averiguaremos si alguno de ellos se combina con el sustantivo acondicionador. Solo hallamos 1 ocurrencia que combina acondicionador con tonificante. Este resultado no puede ser concluyente, aunque sí relaciona claramente la acción anticaída con la propiedad tonificante del producto.

Acondicionador antienvejecimiento y tonificante para todo tipo de cabello con citricos y miel

Realizamos ahora una búsqueda avanzada, para localizar si el adjetivo tonificante se relaciona en alguna ocasión con otro producto capilar anticaída distinto del acondicionador, utilizando tonificante como nodo y caída entre las 10 palabras a la izquierda o a la derecha, con lo que llegamos al siguiente resultado:

CHAMPÚ DE EUCALIPTO -D’Shila-.

Descripción del producto:

Frena la caída del cabello gracias a su acción estimulante y tonificante que refuerza el bulbo piloso.

39 El programa WS permite acceder al texto completo pinchando dos veces en la línea de concordancia que nos interesa. 
Poco a poco, pues, y gracias a sucesivas consultas del corpus, una opción -en este caso tonificante - adquiere más fuerza que las demás.

En otras ocasiones recurriremos al corpus para realizar consultas de tipo sintáctico. Es sabido que muchos de los problemas en el aprendizaje de italiano por parte de españoles o del español por parte de italianos son de tipo sintáctico, pues tienen su origen en divergencias como, por ejemplo, el uso de las preposiciones, la formación de sintagmas, las proposiciones relativas, el uso de los tiempos en las oraciones subordinadas, etc.

En el TO se detecta un predominio claro del estilo nominal debido al empleo de sustantivos y adjetivos derivados de verbos a los que sustituyen en su correspondiente forma personal. Observemos un ejemplo:

3) nello stesso tempo la propoli d'api e il ginseng tonificano e rinforzano i capelli che acquistano luminosità e ottimale pettinabilità evitando il rischio di strappo da pettine

Comprobamos cómo pettinabilità sustituye sintagmas del tipo facili da pettinare y cómo evitando il rischio di strappo da pettine sustituye la frase pasiva (il rischio di) essere strappati dal pettine. Nuestro objetivo ahora es analizar si en los textos que conforman nuestro corpus existe o no la misma tendencia nominal. Para ello extraemos concordancias para el sustantivo equivalente a strappo en español, a saber, rotura.

Hallamos 5 ocurrencias de rotura, algunas de ellas de corte muy parecido a la expresión del TO: evita la rotura del cabello, disminuye la rotura. Con las búsquedas romperse, partirse, partid ${ }^{*}$, roto, no se encuentra ocurrencia alguna, por lo que optamos por mantener el sintagma nominal también en el TT.

\section{CONCLUSIONES}

En este trabajo hemos presentado el corpus como una útil herramienta didáctica en las clases de traducción especializada italiano-español. En concreto, hemos querido ilustrar la aplicación de un corpus virtual construido ad hoc para afrontar con éxito un encargo de traducción de italiano a español de textos procedentes de la industria cosmética.

Una de las competencias fundamentales del traductor de hoy en día es su capacidad para utilizar eficazmente las fuentes de información que tiene a su disposición para alcanzar un resultado final de calidad.

Si, como afirma A. Hurtado (2001: 62), la documentación en la traducción especializada es un pilar fundamental para conocer el campo temático, la terminología y las normas de funcionamiento textual, debemos por fuerza concluir que un corpus bien diseñado y compilado constituye un recurso de inestimable valor que los futuros traductores habrán de conocer y manejar durante su andadura académica y profesional. En el caso de la traducción 
especializada, no solo servirá para adquirir conocimientos sobre el dominio de especialidad en cuestión, sino que también constituirá una guía para que el texto traducido presente un formato y estilo apropiados y para que haga uso de la terminología y fraseología precisas. Hemos podido comprobar cómo el corpus se puede interrogar para obtener información de tipo enciclopédico, discursivotextual, fraseológico, terminológico, estilístico. En nuestro caso, el corpus CCR nos ha permitido resolver problemas de índole terminológica, documental y sintáctico-estilística.

Uno de nuestros fines como docentes de traducción debe ser, por tanto, que los alumnos aprendan a construir corpus específicos para sus necesidades documentales y terminológicas ante un encargo de traducción especializada. No es infrecuente que los estudiantes, incluso en los últimos años de sus estudios, se queden en el paso de una búsqueda general en motores como Google para demostrar, por ejemplo, la validez de un término como equivalente de traducción. Las ventajas de la utilización de corpus en la formación del futuro traductor no residen solo en la posibilidad de resolver con relativa rapidez las dificultades planteadas por el TO, sino que en la propia construcción del corpus, el estudiante ha de trabajar destrezas vitales en su profesión: exhaustivo análisis lingüísticopragmático del TO, conciencia clara del objetivo del encargo, búsqueda selectiva del tipo de documentos requeridos para nutrir el corpus, evaluación de la calidad y fiabilidad de las fuentes encontradas, manejo correcto de programas de gestión de corpus, etc.

Una correcta explotación del corpus no solo sirve al discente para justificar una determinada elección en su traducción, sino que también proporciona al docente argumentos para defender su evaluación de la traducción realizada por el alumno. Por otra parte, no se puede descartar, como señala Aston (2000: 25), que durante la consulta del corpus se produzcan hallazgos inesperados que sean igualmente interesantes para la labor del traductor, al sugerir soluciones o interpretaciones no previstas por este.

En la combinación lingüística italiano/español y en el ámbito de especialidad que hemos seleccionado para el encargo planteado, la construcción de un corpus ad hoc está aún más justificada, pues es clara la inferioridad en el número de recursos lexicográficos disponibles frente a otros campos como la medicina o el derecho en lenguas como inglés, francés o alemán. De ahí que hayamos querido ofrecer asimismo un breve repertorio de recursos impresos y electrónicos que cumplieran ambas condiciones.

Entre los planes futuros que abre esta línea de investigación, destacamos la construcción y utilización de un corpus comparable más extenso para caracterizar el lenguaje de especialidad de la industria cosmética desde un enfoque contrastivo italiano/español, así como para la didáctica de la traducción inversa, ámbito donde también han quedado suficientemente demostradas las virtudes de esta herramienta. 


\section{BIBLIOGRAFÍA}

Alarcón, R., Bach, C., Sierra, G., "Extracción de contextos definitorios en corpus especializados: hacia la elaboración de una herramienta de ayuda terminográfica", Revista Española de Lingüística, 37 (2007), pp. 247-277.

Alonso, A., et al., "La utilización de corpus paralelos alineados en la docencia de la traducción y de los lenguajes de especialidad”, en L. Iglesias - S. Doval (eds.), Studies in Contrastive Linguistics. Proceedings of the Second International Contrastive Linguistics Conference, Santiago de Compostela, Publicacións de la Universidade de Santiago de Compostela, 2001, pp. 71-82.

Alvar Ezquerra, M. - Corpas Pastor, G., "Criterios de diseño para la creación de córpora”, en M. Alvar Ezquerra - J.A. Villena Ponsoda (coords.), 1994, pp. 31-40.

Alvar Ezquerra, M. - Villena Ponsoda, J.A. (coords.), Estudios para un corpus del español, Málaga, Servicio de Publicaciones de la Universidad, 1994.

Alvar Ezquerra, M. - Blanco Rodríguez, V. - Pérez Lagos, F., "Diseño de un corpus español en el marco de un corpus europeo", en M. Alvar Ezquerra - J.A. Villena Ponsoda (coords.), 1994, pp. 9-29.

Aston, G., "Corpora and language teaching”, en L. Burnard - T. McEnery (eds.), 2000, pp. 7-17.

Aston, G., "I corpora come risorse per la traduzione e per l'apprendimento", en S. Bernardini - F. Zanettin (eds.), 2001, pp. 21-30.

BAKER, M.,"Corpus linguistics and Translation Studies-Implications and Applications", en M. Baker - G. Francis - E. Tognini-Bonelli (eds.), Text and Technology. In Honour of John Sinclair, Ámsterdam/Philadelphia, John Benjamins, 1993, pp. 233-252.

BAKer, M., "Corpora in Translation Studies: An Overview and Some Suggestions for Future Research", Target, 7, 2 (1995), pp. 223-243.

BeEby, A.- Rodríguez, P. - Sánchez-Gijón, P. (eds.), Corpus use and translating: corpus use for learning to translate and learning corpus use to translate. Ámsterdam/Philadelphia, John Benjamins, 2009.

Bernardini, S., "Systematising serendipity: Proposals for concordancing large corpora with language learners", en L. Burnard - T. McEnery (eds.), 2000, pp. 225-234.

Bernardini, S. - Zanettin, F., I corpora nella didattica della traduzione - Corpus use and learning to translate. Bolonia, CLUEB, 2000.

Blecua, J.M. - Clavería, G.- SÁnchez y Torruella, J. (eds.), Filología e informática. Nuevas tecnologías en los estudios filológicos. Barcelona, Milenio / Universidad Autónoma de Barcelona, 1999.

BLinI, L., "Aspectos interculturales en la traducción de publicidad: una experiencia didáctica”, en G. Bazzocchi - P. Capanaga (eds.), Mediación lingüística de lenguas afines: español/italiano, Bolonia, Gedit Edizioni, 2006.

Bowker, L. - Pearson, J., Working with Specialized Language: A practical guide to using corpora, Londres, Routledge, 2002.

Burnard, L. - McEnery, T. (eds.), Rethinking Language Pedagogy from a Corpus Perspective, Berna, Peter Lang, 2000.

CabrÉ, M.T., "El Discurs Especialitzat o la Variació Funcional determinada per la Temática”, Caplletra, 25 (1998), pp. 173-194. 
Cabré, M.T., "La terminología en la traducción especializada”, en C. Gonzalo García V. García Yebra (eds.), 2004a, pp. 89-122.

CABré, M.T., “¿Lenguajes especializados o lenguajes para propósitos específicos?”, en A.Van Hooft (dir.), Textos y discursos de especialidad: el español de los negocios, Revista Foro Hispánico, 26, 2004b, pp. 19-34.

Cabré, M.T., "Constituir un corpus de textos de especialidad: condiciones y posibilidades”, en M. Ballard - C. Pineira-Tresmontant (ed.), Les corpus en linguistique et en traductologie, Arras, Artois Presses Université, 2007, pp. 89-106.

Calzolari, N. - Bindi, R., "Acquisition of Lexical Information from a Large Textual Italian Corpus", COLING-90 (Proceedings of the 13th International Conference on Computational Linguistics), Helsinki, 3, 1990, pp. 54-59.

Cardinaletti, A. - Garzone, G., L'italiano delle traduzioni, Milán, FrancoAngeli Edizioni, 2005.

Cardinaletti, A., "Ancora sull'italiano delle traduzioni”, Other Modernities: Rivista di studi letterari e culturali, 2012 http://riviste.unimi.it/index.php/AMonline/article/ view/2462. 10-01-14.

Castillo Rodríguez, C., "La elaboración de un corpus ad hoc paralelo multilingüe", Revista Tradumática: Traducció i Tecnologies de la Informació i la Comunicació, 7 (2009). http://www.fti.uab.cat/tradumatica/revista 10-01-14.

Corpas Pastor, G., "El uso de córpora en traducción e interpretación”, en C. Martín Vide (ed.), Actas del XI Congreso de Lenguajes Naturales y Lenguajes Formales, Barcelona, PPU, 1995, pp. 381-387.

Corpas Pastor, G.,"Compilación de un corpus ad hoc para la enseñanza de la traducción inversa especializada", Trans, 5 (2001), pp. 155-184.

Corpas Pastor, G., “Traducir con corpus: de la teoría a la práctica”, en J. García Palacios - M. T. Fuentes Morán (eds.), Texto, terminología y traducción, Salamanca, Almar, 2002, pp. 189-226.

Corpas Pastor, G., "Localización de recursos y compilación de corpus via internet: aplicaciones para la didáctica de la traducción médica especializada”, en C. Gonzalo García - V. García Yebra (eds.), Manual de documentación y Terminología para la traducción especializada, Madrid, Arco, 2004a, pp. 223-258.

Corpas Pastor, G., "La traducción de textos médicos especializados a través de recursos electrónicos y corpus virtuales”, en L. González - P. Hernúñez (eds.), Las palabras del traductor. Actas del II Congreso Internacional "El español, lengua de traducción”, 20-21 de mayo 2004, Bruselas, Comisión Europea/ESLETRA, 2004b, pp. 137-164. http:// crc.cervantes.es/lengua/esletra/pdf/02/017_corpas.pdf 10-01-14

Corpas Pastor, G., Investigar con corpus en traducción: los retos de un nuevo paradigma, Frankfurt am Main, Peter Lang, 2008.

Corpas Pastor, G. - Seghiri Domínguez, M., El concepto de representatividad en lingüistica de corpus: aproximaciones teóricas y consecuencias para la traducción, Documento Técnico, Departamento de Traducción e Interpretación, Universidad de Málaga., 2006 [BFF2003-04616 MCYT/TI-DT-2006-1].

Díaz Rojo, J.A., "La belleza es salud: la medicalización lingüística de la publicidad de los cosméticos”, Contextos, XIX-XX/37-40 (2001-2002), pp. 109-121. 
Díaz Rojo, J.A. - Morant i Marco, R. - Westall Pixton, D., El Culto a la Salud y Belleza. La retórica del bienestar, Madrid, Biblioteca Nueva, 2006.

Díaz Rojo,J.A. - Morant i Marco, R., "El discurso crítico contra la 'tiranía' del culto al cuerpo", Tonos: Revista electrónica de estudios filológicos, 14 (2007).

http://www.um.es/tonosdigital/znum14/secciones/estudios-8-cuerpo.htm 10-01-14.

Ebeling, J., "Contrastive linguistics, translation, and parallel corpora”, Meta, XLIII, 4 (1998), pp. 1-16.

Elena García, P., "Reflexiones en torno a la enseñanza de la traducción especializada", Panace@, vol. IX, 26 (2007). http://www.tremedica.org/panacea/IndiceGeneral/n26_ editorial.pdf 10-01-14.

Flores Acuña, E., (en prensa), "Lexicografía especializada bilingüe/multilingüe italiano-español y enseñanza de la traducción especializada". Conferencia presentada en el Convegno Norma e uso nella lessicografia bilingue. Ragusa, 18-20 octubre 2012.

Gonzalo García, C. - Fraile Vicente, E., "Selección y evaluación de recursos lingüísticos en internet para el traductor especializado”, en C. Gonzalo García - V. García Yebra (eds.), 2004, pp. 337-360.

Gonzalo García,C.- García Yebra, V.(eds.), Manual de documentacióny Terminología para la traducción especializada, Madrid, Arco, 2004.

Haensch, G., Los diccionarios del español en el siglo XXI: problemas actuales de la lexicografía; los distintos tipos de diccionarios: una guía para usuario; bibliografía de publicaciones sobre lexicografía. Salamanca, Ediciones Universidad de Salamanca, 2004.

Hurtado Albir, A., Traducción y Traductología, Cátedra, Madrid, 2001.

Jensen, V. - Mousten, B. - Laursen, A.L., "Electronic Corpora as Translation Tools: A Solution in Practice", Communication \& Language at work, 1 (2012), pp. 21-33.

Johansson, S., "On the Role of Corpora in Cross-Linguistic Research", en S. Johansson - S. Oksefjell (eds.), 1998, pp. 3-24.

Johansson, S.- Окsefield, S. (eds.), Corpora and Cross-linguistic Research: Theory, Method and Case Studies, Ámsterdam/Atlanta, Rodopi, 1998.

Kenny, D., Lexis and Creativity in Translation. A Corpus-based Study, Manchester, St. Jerome, 2001.

KüBLER, N., “Corpora and LSP translation”, en F. Zanettin - S. Bernardini - D. Stewart (eds.), 2003, pp. 25-42.

Laviosa, S., "The Corpus-based Approach: A New Paradigm in Translation Studies", Meta: Translator's Journal, 43, 4 (1998), pp. 474-479.

MaiA, B., "Training Translators in Terminology and Information Retrieval Using Comparable and Parallel Corpora", en F. Zanettin - S. Bernardini - D. Stewart (eds.), 2003, pp. 43-54.

Martínez de Sousa, J., "La lexicografía especializada del español actual”, en C. Gonzalo García - V. García Yebra (eds.), Manual de documentación y terminología para la traducción especializada, Madrid, Arco Libros, 2004, pp. 173-190.

Mayoral Asensio, R.,"La traducción especializada como operación de documentación", Sendebar: Revista de la Facultad de Traducción e Interpretación, 8-9 (1997-1999), pp. 
137-154. http://www.ugr.es/ rasensio/docs/Trad._Esp._y_Documentacion.pdf 10-01-14.

Mora de Nieva, M.C., "El léxico de la cosmética, los adornos y la vestimenta en un texto español del s. XV", en E. Rojas Mayer (ed.), Léxico e interculturalidad. Nuevas perspectivas, Tucumán, INSIL, 2012.

Morant Marco, R.- Martín, M.A., "Lenguaje y hermosura, un tema lingüístico que perdura”, Revista de Filología Románica, 27 (2010), pp. 263-284.

Olohan, M., "Corpus Linguistics and Translation Studies: Interaction and Reaction", Linguistica Antverpiensia, 1 (2002), pp. 419-429.

Rodríguez Inés, P., Uso de corpus electrónicos en la formación de traductores (inglésespañol), Tesis doctoral, Departamento de Traducción e Interpretación, Universidad Autónoma de Barcelona, 2008.

Seghiri, M., "Metodología protocolizada de compilación de un corpus de seguros de viajes: aspectos de diseño y representatividad “, RLA. Revista de Lingüística Teórica y Aplicada, Concepción (Chile), 49, 2 (2011), pp. 13-30.

Tena Tena, P., "La cosmética áurea a través de mujeres literarias", Lemir, 8 (2004). http://parnaseo.uv.es/lemir/revista/revista8/tena2.htm 10-01-14.

Torruella, J. - Llisterri, J., "Diseño de corpus textuales y orales", en J.M.Blecua G. Clavería - J. Sánchez y Torruella (eds.), 1999, pp. 45-77.

VAldÉs Rodríguez, M. C., La traducción publicitaria: comunicación y cultura, Valencia: Universitat de València; Castellón de la Plana, Publicacions de la Universitat Jaume I; Barcelona, Universitat Autònoma de Barcelona, Servei de Publicacions, 2004.

VArgas Sierra, C., "Diseño de un corpus especializado con fines terminográficos: el corpus de la piedra natural", Debate Terminológico, 2, 7 (2006), París: RITERM (Red Iberoamericana de Terminología). http://rua.ua.es/dspace/bitstream/10045/9426/1/ chelo_vargas_sierra.pdf 10-01-14.

Ventura, L., La tiranía de la belleza. Las mujeres ante los modelos estéticos, Barcelona, Plaza y Janés, 2000.

Vivaldi Palatresi, J., "Catálogo de herramientas informáticas relacionadas con la creación, gestión y explotación de corpus textuales”, Revista Tradumàtica, 7 (2009). http://webs2002.uab.es/tradumatica/revista/num7/articles/10/10.pdf 10-01-14.

Wilkinson, M., "Using a Specialized Corpus to Improve Translation Quality", Translation Journal, 9, 3 (2005). http://www.translationdirectory.com/article545.htm 10-01-14.

Zanettin, F., "Bilingual Comparable Corpora and the Training of Translators", Meta, 43, 4 (1998), pp. 616-630.

Zanettin, F., "Swimming in words: Corpora, translation and language learning", en G. Aston (ed.), Learning with corpora, Bolonia, CLUEB, 2001, pp. 177-197.

Zanettin, F. - Bernardini, S. - Stewart, D., Corpora in Translator Education, Manchester, St. Jerome, 2003. 


\section{FUENTES LEXICOGRÁFICAS}

Monolingües (ESP)

AA.VV., La cosmética de la A a la Z, Barcelona, Hibiscus, 1998, 88 pp.

AA.VV., Diccionario de Cosmetología, Madrid, Paraninfo, 2000, 312 pp. (Traducción de A. Rincón Córcoles. Título original: Cosmetology dictionary).

AA.VV., Diccionario cosmetológico de la esteticista, Barcelona, Albert d'Arnal, 2003, 115 pp.

Carrasco, F., Diccionario de ingredientes cosméticos, 2009 (4a ed.) www.imagenpersonal. net 10-01-14.

de Castro, M. S., Diccionario breve de estética, Barcelona, MB: Vida estética, 171 pp.

Terrón González,J., Léxico de cosméticos y afeites en el Siglo de Oro, Cáceres, Universidad de Extremadura, Servicio de Publicaciones, 1990, 233 pp.

Monolingües (IT)

AA.VV., Curiamoci con le erbe. Dizionario, cosmesi e dietetica, Milán, Librex. Vol. I: Storia dell'erboristeria e curiamoci con le erbe. Vol. II: Dizionario. Vol. III: Cosmesi e dietetica, 1971.

Bovero, A., Dermocosmetologia. Dall'inestetismo al trattamento cosmetico, Milán, Tecniche nuove, 2011.

De Salvia Baldini, M.S., Dizionario di bellezza: l'arte della cosmesi, Milán, Garzanti, 1995.

Proserpio, G.- Racchini, E., Dizionario di cosmetologia. Mille termini cosmetici dalla A alla Z, Milán, BCM, 2009.

Multilingües

CARRIERE, G., Detergents: a glossary of terms used in the detergents industry, Ámsterdam, Elsevier, 1960.

CARRIERE, G., Elsevier's lexicon of detergents, cosmetics and toiletries, Ámsterdam, Elsevier, 1966.

CArriere, G. - Kiкolski, P., Dictionary of surface active agents, cosmetics, and toiletries. English, French, German, Spanish, Italian, Dutch, Polish, Ámsterdam, Elsevier, 1978. 